\title{
Specificity of learning through memory retrieval practice: The case of addition and subtraction
}

\author{
Daniel Bajic • Jung Kwak • Timothy C. Rickard
}

Published online: 27 August 2011

(C) Psychonomic Society, Inc. 2011

\begin{abstract}
The identical elements (IE) model (Rickard, Healy, \& Bourne, Learning, Memory, and Cognition 32:734-748, 1994) of fact representation predicts that, in both verbal and numerical domains, performance gains with retrieval practice on multielement items will be specific to the practiced stimulus-response combinations, failing to transfer even to altered stimulus-response mappings of practiced items. In the case of arithmetic, the model predicts no transfer across either complementary operations (e.g., $4 \times 7$ to $28 / 4$ ) or complementary division or subtraction problems (e.g., $28 / 4$ to $28 / 7$ ). Although that model has successfully described transfer effects in the domains of multiplication-division and episodic cued recall, it is challenged by a recent demonstration of positive cross-operation transfer for addition and subtraction (Campbell \& Agnew, Psychonomic Bulletin \& Review 16:938-944, 2009). We report results of a new additionsubtraction transfer experiment, the design of which closely matched that of a prior multiplication-division experiment that supported the model. The transfer results were consistent with the IE model. A two-component model of memory retrieval practice effects is proposed to account for the discrepant experimental results for addition and subtraction and to guide future work.
\end{abstract}

Keywords Memory - Learning · Transfer - Identical elements model $\cdot$ Arithmetic

When teaching or practicing any skilled activity, there is typically a hope - if not an expectation - that the learner's

D. Bajic $\cdot$ J. Kwak $\cdot$ T. C. Rickard $(\bowtie)$

Department of Psychology, University of California, San Diego, La Jolla, CA 92093-0109, USA

e-mail: trickard@ucsd.edu burgeoning skills will generalize beyond the specific focus of practice. If, for example, a teacher assigns students a particular set of arithmetic problems, it is likely intended that this practice will result in greater fluency for related arithmetic problems encountered later. But to what extent is this true? Investigations into the specificity of learning that occurs through memory retrieval practice can yield insights into this question, revealing general principles of memory representation and addressing the viability of candidate models of memory-based skills.

For the cases of cued episodic recall practice (Rickard \& Bajic, 2006) and multiplication-division, the data support the identical elements (IE) model (Rickard, Healy, \& Bourne, 1994). That model assumes that, regardless of the content domain or the manner in which memory is initially encoded, retrieval practice always yields a separate and independent representation for each unique combination of practiced stimulus elements, ignoring perceptual factors such as stimulus modality and the spatial or temporal ordering of the elements. Each stimulus representation, in turn, has an association to the required response.

The set of representations that is predicted by the IE model is shown for sample multiplication-division $(4,7$, $28)$ and addition-subtraction $(4,7,11)$ number triplets in Fig. 1. The model assumes that practice on either $4 \times 7$ or $7 \times 4$ strengthens (i.e., facilitates subsequent access of) only the multiplication representation in Fig. 1 and that practice on either division problem strengthens only its corresponding representation. Analogous assumptions hold for addition-subtraction.

In the original version of the IE model, corresponding to Fig. 1 without the left-going arrow for multiplication, only the representation that exactly matches a presented problem (within the limits specified above) can be used successfully to retrieve the answer. Improvements in retrieval performance with practice, therefore, should not transfer to problems whose 

$(4,7, x) \stackrel{\rightarrow}{\leftarrow} 28$
$(4,7,+) \rightarrow 11$
$28 \div 4 \rightarrow 7$
$(11-4) \rightarrow 7$
$28 \div 7 \rightarrow 4$
$(11-7) \rightarrow 4$

Fig. 1 Representations and associations assumed by the IE model for multiplication-division and addition-subtraction. The notations $(4,7, \times)$ and $(4,7,+)$ represent the assumption that the two operand orders for multiplication and addition activate the same representation. The leftgoing arrow for multiplication was incorporated into a revised version of the model (Rickard, 2005)

elements do not match the practiced representation, even if the same number triplet is involved. Speedup with retrieval practice on $4 \times 7$ should not transfer to $28 \div 4$, and vice versa. Furthermore, speedup on $28 \div 4$ should not transfer to $28 \div 7$, and vice versa. Speedup on $4 \times 7$ will, however, transfer to $7 \times 4$ (and vice versa). Analogous predictions apply for addition-subtraction.

For multiplication-division, the IE predictions have, to a close approximation, held across several experimental paradigms (for reviews, see Campbell \& Agnew, 2009; Rickard, 2005). The relatively small cross-operation transfer that has sometimes been observed-primarily for large division problems (e.g., LeFevre \& Morris, 1999) — can be accommodated by a revised version of the model (Rickard, 2005) in which multiplication practice simultaneously strengthens bidirectional associations, as depicted by the combined right- and left-going arrows for multiplication in Fig. 1 (see also Campbell \& Robert, 2008; Rusconi, Galfano, Rebonato, \& Umiltà, 2006). At intermediate skill levels, wherein the IE associations for large division problems may not yet have formed, the reverse (i.e., left-going) association from the product back to the corresponding multiplication operands may support a division-by-factoring strategy that yields positive transfer between operations (for details, see Rickard, 2005). At higher skill levels, however, the full set of IE associations has been learned, and direct retrieval through the division associations is selected in lieu of the slower and more complex division-by-factoring strategy. From that point onward, Rickard proposed, the simpler version of the IE model (with no left-going association in Fig. 1) holds, and cross-operation transfer should not occur. Consistent with that prediction, cross-operation transfer has not been observed for small problems (e.g., $3 \times 4$ to $12 \div 3$ ), for which adult performance is most likely to reflect memory retrieval (Zbrodoff \& Logan, 2005).

Unlike multiplication, each addition sum can result from multiple operand combinations, rendering useless any association from the sum to the problem operands (as would be depicted in Fig. 1 by a left-going arrow for addition; see Rickard, 2005; Campbell \& Robert, 2008).
Thus, at intermediate skill levels, the IE prediction of no cross-operation transfer is stronger for addition-subtraction than it is for multiplication-division. Although there is some empirical support for that prediction (e.g., Campbell, FuchsLacelle, \& Phenix, 2006), more recent demonstrations of substantial cross-operation transfer between addition and subtraction (Campbell \& Agnew, 2009; see also Campbell \& Alberts, 2010) are problematic for the IE model.

In the Campbell and Agnew (2009) experiment, subjects were trained on 12 addition and 12 subtraction problems, with 1 problem being derived from each of 24 number triplets. Half of the subjects were then given a transfer test on 24 addition problems derived from the 24 trained triplets, plus 12 addition problems derived from 12 untrained triplets. The remaining subjects were given a transfer test on 24 subtraction problems derived from the 24 trained triplets, plus 12 subtraction problems derived from 12 untrained triplets. Thus, all subjects encountered three transfer conditions: a nochange condition, an operation change condition, and a new problems condition. In contrast with the IE predictions, response times (RTs) were significantly shorter for operation change problems than for new problems.

Campbell and Agnew (2009) concluded that the IE model does not provide a sufficient account for the case of addition-subtraction. They suggested two possible explanations of the difference between their results and the aforementioned results for multiplication-division. First, for addition-subtraction, fact organization may take the form of a holistic representation for each number triplet. If training on a specific problem speeds subsequent access to the holistic triplet representation in a manner that is accessible to all problems derivable from that triplet, cross-operation transfer will be observed (for related models, see Anderson, Fincham, \& Douglass, 1997; Rabinowitz \& Goldberg, 1995). This account leaves unaddressed the question of why holistic triplet representations apparently do not exist for or, at least, are not a significant factor underlying performance on multiplication-division.

Second, Campbell and Agnew (2009) suggested that addition-subtraction may be performed by way of semantic quantity (e.g., mental number line) procedures, rather than by retrieval via discrete fact representations. If training makes subjects generally more efficient with these procedures in the particular number line regions that correspond to the trained problems, positive transfer to operation change problems might be observed. Note that analogous mental number line operations cannot underlie multiplicationdivision performance (barring the possibility that subjects perform logarithmically scaled mental "slide-rule" calculations for those operations).

In the experiment reported below, we attempted to replicate the Campbell and Agnew (2009) findings for addition-subtraction, but using a design that closely 
matched a multiplication-division experiment conducted by Rickard and Bourne (1996, Experiment 1). The design differs from Campbell and Agnew's in multiple respects, including response modality, transfer conditions investigated, mixing versus not mixing of operations within each block, time interval between trials, amount of practice, presence or absence of strategy probing, number of training sessions, and the delay between the training and transfer tests.

If the results replicate those of Campbell and Agnew (2009), cross-operation transfer for addition-subtraction will have proven robust across disparate experimental paradigms, and Campbell and Agnew's proposals for addition-subtraction representation will be supported. Alternatively, the results may be as predicted by the IE model. That outcome would raise the intriguing possibility that, for arithmetic and perhaps other domains, the specificity of learned representations depends critically on the implementation of the training regimen.

\section{Method}

\section{Subjects}

Twenty-four University of California at San Diego undergraduate students participated for course credit.

Materials, design, and procedure

Subjects were tested individually on personal computers. The experiment consisted of three sessions over 5 days. Stimuli were visually presented addition and subtraction problems constructed from three sets of eight additionsubtraction number triplets (see Table 1). From those three sets, 96 distinct arithmetic problems can be formed (2 addition and 2 subtraction problems from each triplet). For the triplet $5,7,12$, for example, the 4 problems are $5+7,7+5$, $12-7$, and $12-5$. In session 3 (the transfer test), the full set of 96 problems was presented. In sessions 1 and 2, each subject received training on 16 problems, consisting of 8 addition problems derived from one of the sets of eight triplets in Table 1 and 8 subtraction problems derived from another of

Table 1 The three triplet sets

\begin{tabular}{lll}
\hline Set 1 & Set 2 & Set 3 \\
\hline $3,5,8$ & $5,4,9$ & $3,7,10$ \\
$9,2,11$ & $2,6,8$ & $4,3,7$ \\
$4,6,10$ & $6,5,11$ & $2,8,10$ \\
$7,5,12$ & $3,6,9$ & $3,8,11$ \\
$6,7,13$ & $7,8,15$ & $8,5,13$ \\
$9,8,17$ & $9,3,12$ & $5,9,14$ \\
$4,9,13$ & $6,8,14$ & $7,4,11$ \\
$9,7,16$ & $8,4,12$ & $9,6,15$ \\
\hline
\end{tabular}

the sets of eight triplets. One set of eight triplets was withheld from training to serve as new problems on the transfer test. Full counterbalancing of which two triplet sets were used to derive the training problems and of the four possible problem types (i.e., addition or subtraction; ascending or descending operand order) yielded 12 sets of 16 training problems. In each set of training problems, half of the problems had ascending left-to-right operand order, and half had descending order. Two subjects were trained on each set.

For subtraction problems on the transfer test, all eight single-digit keypress responses were required for problems in both the new problems condition and the combined operation and operation plus order change conditions. Analogously, for addition, exactly three of the eight twodigit keypress answers in both the new problems condition and the combined operation and operation plus order change conditions were answers to (different) training problems. Thus, any motor-stage latency facilitation that might occur during training for specific required keypress responses would be unlikely to differentially influence RTs in the pivotal comparisons among those conditions.

Within each session, a block was defined as one randomly ordered presentation of each of that session's problem stimuli, yielding 16 trials per block in sessions 1 and 2 and 96 trials per block in session 3. In session 3, problems derived from the same triplet were not presented on consecutive trials. Subjects received 40 blocks in session 1, 50 in session 2, and 5 in session 3 .

Subjects were instructed to work as quickly and accurately as possible while continuously trying to improve their performance. All trials consisted of (1) a 500-ms blank screen, (2) presentation of a stimulus (e.g., " $6+7=$ " or "13 - 7 = -) at the center of the screen, and (3) the subject's response, typed on the computer's number keypad. If the subject entered an incorrect response, the word "Incorrect" was displayed, along with the correct answer just below it, for 1,500 ms.

At the start of each block, the current block number and the message "Get ready" were displayed for $1 \mathrm{~s}$. At the end of each block, the subject's accuracy percentage and mean $\mathrm{RT}$ on that block were presented for $2 \mathrm{~s}$. When ready, the subject pressed a key to proceed to the next block.

\section{Results \\ Training \\ On the first training block, the error rate was .052 for addition and .036 for subtraction. On the 90th block, these values were .042 and .073 , respectively. \\ All reported RT results are for keypress initiation latency (i.e., latency from stimulus presentation to the first keypress).}


Analyses in which RT was measured to the second keypress (for items with two-digit answers) yielded equivalent results. The RT analyses discussed below were performed on the full set of correctly answered problems. Supplementary analyses limited to "easy problems" (i.e., the first four problems listed in each set of eight in Table 1) and "difficult problems" (the remaining four problems in each set of eight) yielded equivalent results. To minimize outlier influences, log transformation of each trial-level RT was performed prior to analysis. Antilogs of the mean log RTs are presented in the figures.

Mean RTs are shown in Fig. 2 as a function of block and arithmetic operation. The RT convergence by the end of training mirrors that observed for multiplication and division by Rickard and Bourne (1996). The combined RT and error data suggest a speed-accuracy trade-off, such that, for subtraction, there is greater accuracy emphasis at the beginning of training but greater speed emphasis toward the end of training.

\section{Transfer test: addition}

Example problems for the addition transfer test are shown in the top half of Table 2, along with the IE predictions.

Response time Mean RTs are plotted in the top panel of Fig. 3 as a function of block and condition. To efficiently test the IE predictions, four orthogonal transfer condition contrasts were performed. Each contrast involved a withinsubjects factorial analysis of variance with factors of block, transfer condition, and their interaction. A description of each contrast, along with the statistical results for the condition and the condition $\times$ block interaction factors, is given in Table 3. The effect of block was significant in most

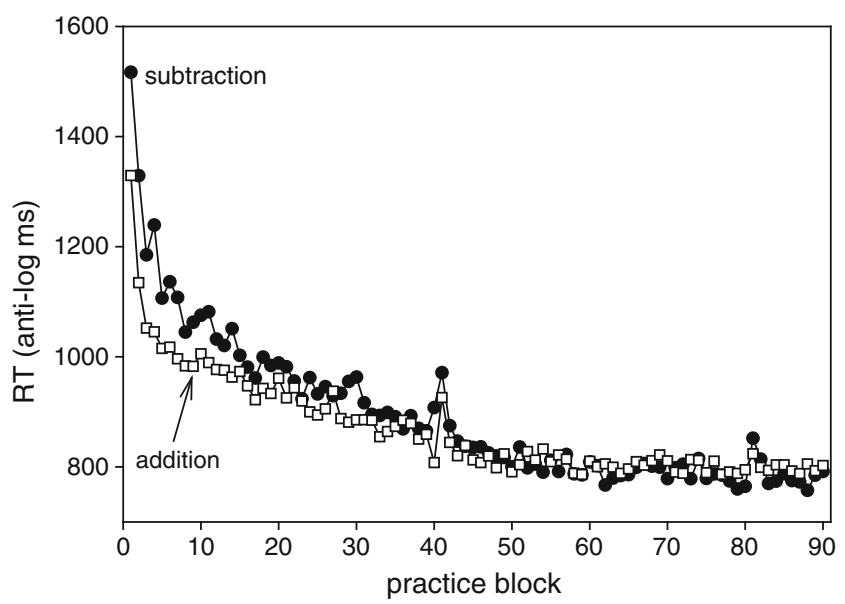

Fig. 2 Antilog of the mean $\log$ response times (RTs) as a function of operation and training block cases, confirming the expected continuation of learning across transfer blocks. Note that supplemental contrasts limited to the first transfer block, which provides the purest measure of transfer from training, replicated the following pattern with respect to the condition effects.

The contrast comparing the RTs in the no-change and order change conditions with those in the other three conditions (contrast 1) was highly significant. The interaction between block and condition was also highly significant, reflecting the fact that the conditions being compared were at different points on the inherently nonlinear RT speedup curve. In contrast, neither the condition factor nor the condition $\times$ block interaction approached significance in the contrasts limited to the operation change, operation plus order change, and new problems conditions (contrasts 2 and 3 ).

The roughly 80 -ms difference between the no-change and order change conditions was highly significant (contrast 4). This effect, which was previously observed for multiplicationdivision, may reflect learning or strengthening during training of specific element configurations corresponding to no-change problems (e.g., a visual memory may form for the configuration " $4 \times 7$ "). Assuming that such representations can become part of the input to the IE-based answer retrieval, the RT advantage for no-change or order change problems can be accommodated by that model.

To evaluate the relative evidence for the null and alternative hypotheses, JZS Bayes factors (Rouder \& Morey, 2011; henceforth, $J Z S-B F$ ) were calculated for the paired $t$-tests that correspond to the four addition single degree of freedom (i.e., main effect) contrasts listed in Table $3 .{ }^{1}$ The $J Z S-B F$ results were $<0.0001,6.22,4.57$, and $<0.001$ for contrasts $1-4$, respectively. These values correspond, using the widely accepted categorization scheme (e.g., Wagenmakers, Wetzels, Borsboom, \& van der Maas, 2011), to extreme evidence for $\mathrm{H}_{1}$, substantial evidence for $\mathrm{H}_{0}$, substantial evidence for $\mathrm{H}_{0}$, and extreme evidence for $\mathrm{H}_{1}$, respectively.

Error rates Error rates (bottom panel of Fig. 3) were more variable than were RTs and, thus, were averaged over blocks prior to analysis. The equivalent of contrast 1 in Table 3 was highly significant [sign test on the subject-level error difference scores, $p<.0001 ; t(23)=5.67]$. There were no trends toward significant error rate differences for contrasts 2-4. The corresponding JZS-BFs, based on paired $t$-tests, were $<0.001$ (extreme $\mathrm{H}_{1}$ ), 5.40 (substantial $\mathrm{H}_{0}$ ), 5.67 (substantial $\mathrm{H}_{0}$ ), and 4.55 (substantial $\mathrm{H}_{0}$ ), for contrasts $1-4$, respectively.

\footnotetext{
${ }^{1}$ Bayes factors were computed using an online calculator for paired $t$ tests (http://pcl.missouri.edu/bayesfactor)
} 
Table 2 Test conditions for an example problem

\begin{tabular}{|c|c|c|c|}
\hline Test condition & Training & Test & IE Prediction \\
\hline \multicolumn{4}{|c|}{ Addition } \\
\hline No change & $3+7=$ & $3+7=$ & reference condition for full transfer \\
\hline Order change & $7+3=$ & $3+7=$ & strong transfer \\
\hline Operation change & $10-3=$ & $3+7=$ & no transfer \\
\hline Order and operation change & $10-7=$ & $3+7=$ & no transfer \\
\hline New problems & & $3+7=$ & reference condition for no transfer \\
\hline \multicolumn{4}{|c|}{ Subtraction } \\
\hline No change & $10-3=$ & $10-3=$ & reference condition for füll transfer \\
\hline Order change & $10-7=$ & $10-3=$ & no transfer \\
\hline Operation change & $3+7=$ & $10-3=$ & no transfer \\
\hline Order and operation change & $7+3=$ & $10-3=$ & no transfer \\
\hline New problems & & $10-3=$ & reference condition for no transfer \\
\hline
\end{tabular}

Transfer test: subtraction

Response time Mean RTs are shown in the upper panel of Fig. 4. Contrast 1 (see Table 3) was highly significant, as was the corresponding interaction. None of the other contrast

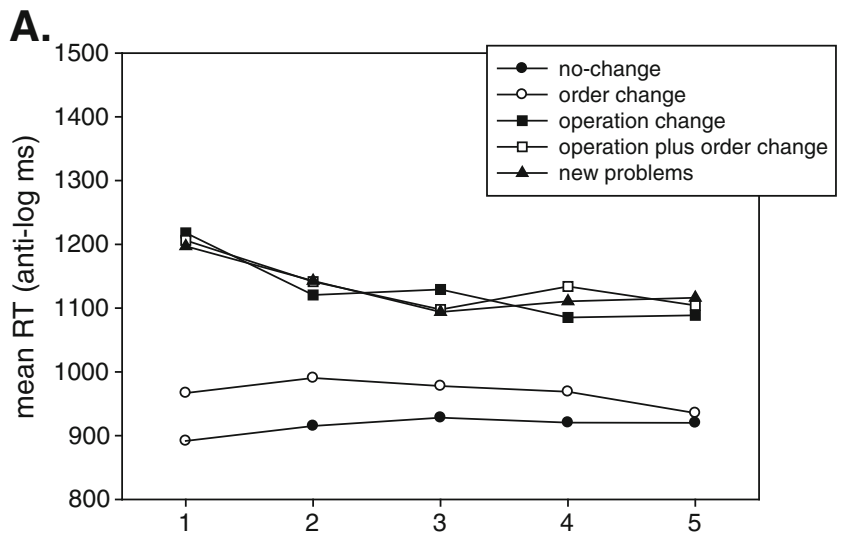

B.

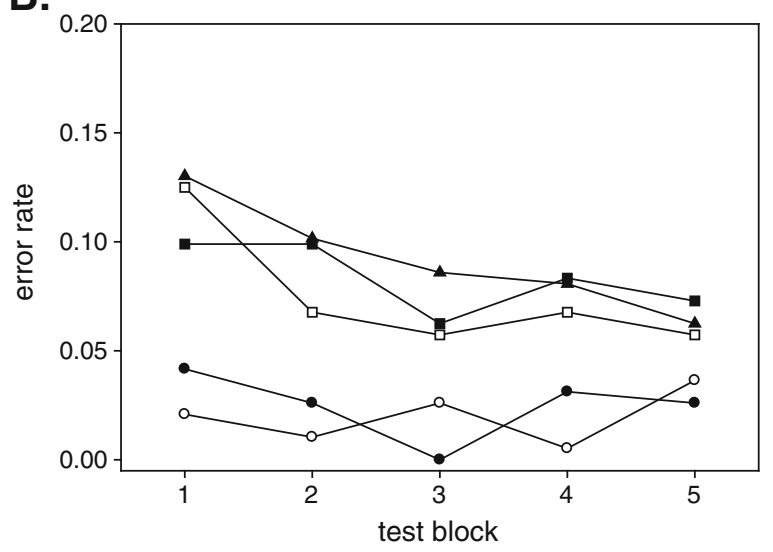

Fig. 3 Addition performance on the transfer test as a function of block and transfer condition. a Antilog of the mean log response times (RTs). b Accuracy effects approached significance. Supplemental RT analyses limited to the first transfer block produced a highly significant effect for contrast $1(p<.0001)$ and a marginally significant effect for contrast $3(p=.049 ; J Z S-B F=0.92$, anecdotal for $\left.\mathrm{H}_{1}\right)$. There were no trends toward significance for the other first block contrasts. $J Z S-B F S$ for the main effect contrasts listed in Table 3 (i.e., averaging over the five test blocks) were $<0.0001$ (extreme $\mathrm{H}_{1}$ ), 4.69 (substantial $\mathrm{H}_{0}$ ), 2.76 (anecdotal $\mathrm{H}_{0}$ ), and 5.06 (substantial $\mathrm{H}_{0}$ ).

Error rates The equivalent of subtraction contrast 1 (Table 3 ) on the error rates averaged over transfer blocks was significant [sign test on the difference scores, $p<.003$, $t(23)=4.86]$, an effect that is visually most evident on the first block. None of the other three contrasts were significant by the sign test, although contrast 3 was marginally significant by the paired $t$-test, $\mathrm{t}(23)=-2.11$. $J Z S-B F S$ for the four contrasts were 0.0038 (extreme $\mathrm{H}_{1}$ ), 3.46 (substantial $\mathrm{H}_{0}$ ), 0.89 (anecdotal $\mathrm{H}_{1}$ ), and 1.76 (anecdotal $\mathrm{H}_{0}$ ).

Curiously, error rates for the operation plus order change condition, but not for the other conditions, decreased systematically over blocks. A post hoc $t$-test comparing the mean error rate (averaged over blocks) in that condition with that of the new problems condition was significant, $t(23)=-2.48$. The evidence for $\mathrm{H}_{1}$ in that test, however, is only anecdotal $(J Z S-B F=0.46)$.

\section{Discussion}

Among the 17 main effect contrasts (i.e., condition effects averaged over test blocks) that were conducted on RTs and errors, the IE model predicted that 5 would be statistically significant. All 5 of those contrasts were, in fact, highly 
Table 3 Analysis of variance results

\section{Conditions Contrasted Condition Effect Interaction}

\begin{tabular}{llll}
\hline Addition & & & \\
Contrast 1 & $1 \& 2$ vs. $3,4, \& 5$ & $F(1,23)=44.8, p<.0001$ & $F(4,92)=5.88, p<.001$ \\
Contrast 2 & $3 \& 4$ vs. 5 & $F(1,23)=0.05$, n.s. & $F(4,92)=0.77$, n.s. \\
Contrast 3 & 3 vs. 4 & $F(1,23)=0.70$, n.s. & $F(4,92)=0.73$, n.s. \\
Contrast 4 & 1 vs. 2 & $F(1,23)=38.6, p<.0001$ & $F(4,92)=1.43$, n.s. \\
Subtraction & & & \\
Contrast 1 & 1 vs. $2,3,4, \& 5$ & $F(1,23)=171.59, p<.0001$ & $F(4,92)=5.96, p<.001$ \\
Contrast 2 & $2,3 \& 4$ vs. 5 & $F(1,23)=0.64$, n.s. & $F(4,92)=0.47$, n.s. \\
Contrast 3 & 2 vs. $3 \& 4$ & $F(1,23)=1.82$, n.s. & $F(4,92)=1.01$, n.s. \\
Contrast 4 & 3 vs. 4 & $F(1,23)=0.48$, n.s. & $F(4,92)=0.63$, n.s. \\
\hline
\end{tabular}

significant ( $p$-values $<.0027$, and extreme evidence for $\mathrm{H}_{1}$ in all cases based on $J Z S-B F S$ ), and all 5 remained significant even when the conservative Bonferroni correction for 17 comparisons (yielding $\alpha=.0029$ ) was applied. Among the remaining 12 main effect contrasts, for which the IE model predicts null results, $J Z S-B F$ evidence was substantial for $\mathrm{H}_{0}$ in 8 , anecdotal for $\mathrm{H}_{0}$ in 2 , and anecdotal for $\mathrm{H}_{1}$ in 2 (1 of which was conducted post hoc).
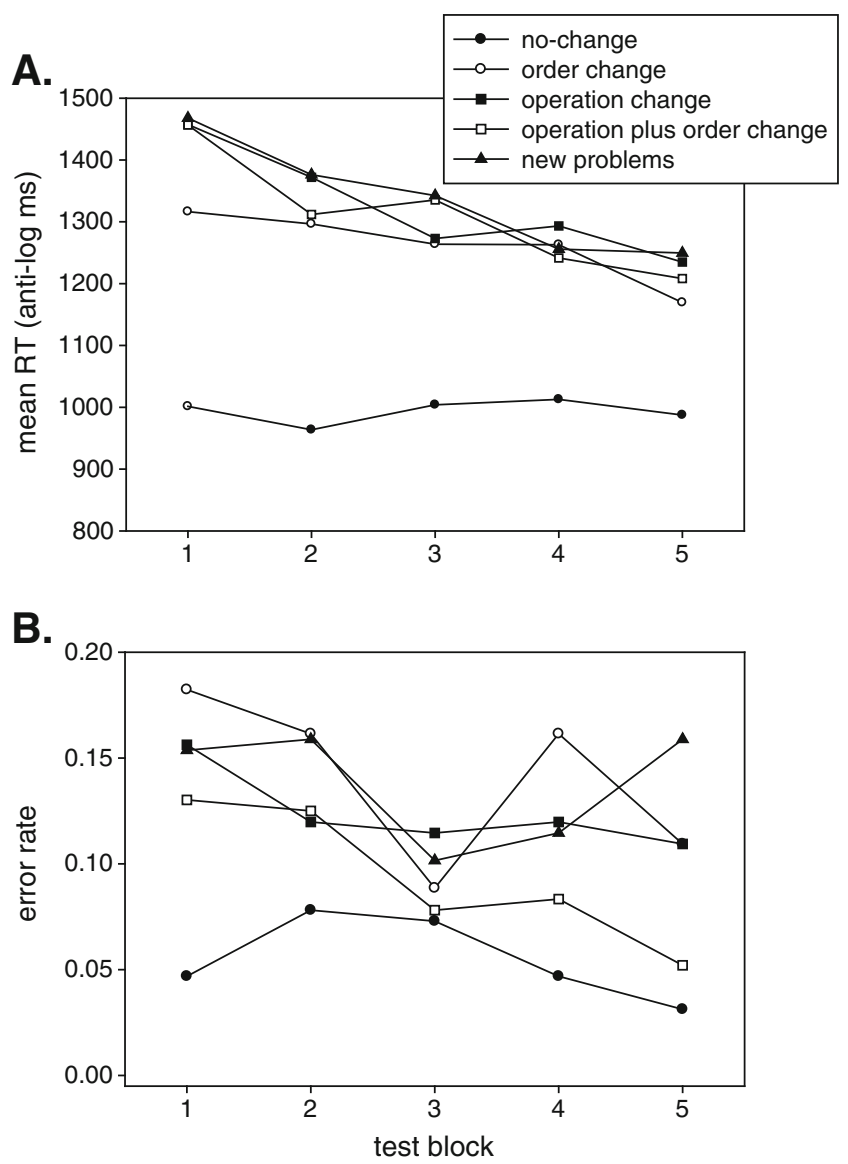

Fig. 4 Subtraction performance on the transfer test as a function of block and transfer condition. a Antilog of the mean log response times (RTs). b Accuracy
In combination, this experiment and Rickard and Bourne's (1996) Experiment 1 constitute the first procedurally matched investigation of transfer for the two complement pairs of arithmetic operations: addition-subtraction and multiplication-division. The finding of nearly identical transfer patterns in the two experiments has two important implications for adult arithmetic representation. First, whatever form that representation may have, it appears to be identical or at least highly similar for the two complement pairs. Second, mental number line procedures, which do not plausibly underlie multiplication-division performance, are apparently also not central to adult addition-subtraction. We cannot strictly rule out an alternative account in which mental number line procedures underlie addition-subtraction performance and in which training on those procedures yields transfer results that happen to exactly match the IE predictions. Parsimony, however, favors the former account.

An important goal for future work is identification of the factors that underlie the diverging transfer results of the present experiment, as compared with those in Campbell and Agnew (2009). As was noted in the introduction, there are multiple differences between the two experiments with respect to both overall design and trial-level procedures. An intriguing possibility is that the specificity and transferability of learning - in arithmetic and, perhaps, other domainsdepends critically on currently undocumented properties of training regimens.

Multiple experiments will likely be needed to identify which of the design differences critically drive the observed transfer differences. Here, we attempt to provide guidance by advancing a candidate theoretical framework. The framework, which builds on a dual-memory model proposed by Rickard and Bajic (2006), has three core assumptions. (1) There are distinct IE and episodic memory representations that can support arithmetic fact retrieval. The IE representations are strengthened through retrieval practice and are the basis for most retrieval speedup. Episodic memory, in contrast, is memory for specific trials, and it is assumed in this framework to have a holistic form that can support transfer. 
This flexibility of use, however, comes at the cost of slower retrieval, as compared with strong IE memories. (2) Strengthening of the IE associations is assumed to be obligatory and automatic and to occur simultaneously with, and as a consequence of, retrieval performance. In contrast, episodic memory is assumed to form, in part at least, as a consequence of postresponse rumination about the justsolved problem. (3) The forgetting rate is assumed to be greater for the episodic than for the IE memory components (for a related proposal, see Clawson, Healy, Ericsson, \& Bourne, 2001).

On some transfer trials, particularly those of the operation change, operation plus order change, new problems, and division order change conditions, the IE associations may not support rapid retrieval, due to incomplete prior learning, forgetting, or other random factors that may transiently affect its availability. On such test trials, subjects can either resort to a time-consuming calculation strategy (LeFevre \& Morris, 1999) or attempt retrieval through episodic memory of training trials. Episodic retrieval - although, by our assumption, slower, on average, than IE retrieval-may occur faster than multistep calculation and, if so, would be preferred if IE memory for the item is not available.

Consider, for example, an operation change problem like $15-8$ on a transfer test. If the IE association cannot be accessed for that problem on a particular trial of the transfer test, the subject may use the presented problem as a cue to attempt retrieval of a holistic, episodic memory that may have been encoded during training (i.e., $8+7=15$ ). If successful, that retrieval would include the answer (i.e., 7), avoiding the need for slow calculations on that trial and yielding cross-operation transfer. Retrieval in this case can be said to be "episodically mediated."

This dual-memory model suggests that one or more features of the Campbell and Agnew (2009) design increased the relative influence of the hypothesized episodic memory component, giving rise to cross-operation transfer. Three aspects of their design seem most pertinent. First, unlike the present experiment and those in Rickard and Bourne (1996), their transfer test occurred immediately after the last training block. If, as we have proposed, the episodic memory component is quickly forgotten, an immediate transfer test would facilitate episodically based cross-operation transfer.

Second, the interval between vocal response onset and presentation of the next problem in the Campbell and Agnew (2009) experiment was on the order of several seconds. That design feature may promote postresponse rumination on training trials about the just-solved problem, facilitating formation of episodic memory that can later support cross-operation transfer. The opportunity for postresponse rumination was substantially reduced in the present experiment and in Rickard and Bourne (1996) by the brief delay between trials.
Third, Campbell and Agnew (2009) used strategy probing on the transfer test. Although strategy probing can provide important data, it also carries the risk of reactivity, especially when the strategy categories are potentially leading (Ericsson \& Simon, 1993). One of the strategy categories from Campbell and Agnew was "ADDITION/SUBTRACTION REFERENCE: You used knowledge of the inverse addition or subtraction problem." Presentation of this option may have promoted a strategy - namely, search of episodic memory from the training session for operation complements that may have been presented - that subjects might have otherwise been less likely to employ. The search for an episodic memory from the training sessions could succeed for operation change problems, but by definition, it could not succeed for new problems, potentially yielding an episodically mediated crossoperation transfer effect.

Although this dual-memory account is speculative, it is plausible, and it connects with the more general memory and skill literature. It also provides a theoretically grounded reference point for hypothesis generation and further empirical work.

Author Note Daniel Bajic, Department of Psychology, University of California, San Diego; Jung Kwak, University of California, San Diego; Timothy C. Rickard, Department of Psychology, University of California, San Diego.

\section{References}

Anderson, J. R., Fincham, J. M., \& Douglass, S. (1997). The role of examples and rules in the acquisition of a cognitive skill. Journal of Experimental Psychology. Learning, Memory, and Cognition, 23, 923-945.

Campbell, J. I. D., \& Agnew, H. (2009). Retrieval savings with nonidentical elements: The case of simple addition and subtraction. Psychonomic Bulletin \& Review, 16, 938-944.

Campbell, J. I. D., \& Alberts, N. M. (2010). Inverse reference in adultsElementary arithmetic. Canadian Journal of Experimental Psychology, 64, 77-85.

Campbell, J. I. D., Fuchs-Lacelle, S., \& Phenix, T. L. (2006). Identical elements model of arithmetic memory: Extension to addition and subtraction. Memory \& Cognition, 34, 633-647.

Campbell, J. I. D., \& Robert, N. D. (2008). Bidirectional associations in multiplication memory: Conditions of negative and positive transfer. Journal of Experimental Psychology. Learning, Memory, and Cognition, 34, 546-555.

Clawson, D. M., Healy, A. F., Ericsson, K. A., \& Bourne, L. E., Jr. (2001). Retention and transfer of Morse code reception skill by novices: Part-whole training. Journal of Experimental Psychology. Applied, 7, 129-142.

Ericsson, K. A., \& Simon, H. A. (1993). Protocol analysis: Verbal reports as data. Cambridge, MA: MIT Press.

LeFevre, J., \& Morris, J. (1999). More on the relation between division and multiplication in simple arithmetic: Evidence for mediation of division solutions via multiplication. Memory \& Cognition, 27, 803-812.

Rabinowitz, M., \& Goldberg, N. (1995). Evaluating the structureprocess hypothesis. In F. Weinert \& W. Schneider (Eds.), Memory 
performance and competencies: Issues in growth and development (pp. 225-242). Hillsdale, NJ: Erlbaum.

Rickard, T. C. (2005). A revised identical elements model of arithmetic fact representation. Journal of Experimental Psychology. Learning, Memory, and Cognition, 31, 250-257.

Rickard, T. C., \& Bajic, D. (2006). Cued recall from image and sentence memory: A shift from episodic to identical elements representation. Journal of Experimental Psychology. Learning, Memory, and Cognition, 32, 734-748.

Rickard, T. C., \& Bourne, L. E., Jr. (1996). Some tests of an identical elements model of basic arithmetic skills. Journal of Experimental Psychology. Learning, Memory, and Cognition, 22, 1281-1295.

Rickard, T. C., Healy, A. F., \& Bourne, L. E., Jr. (1994). On the cognitive structure of basic arithmetic skills: Operation, order, and symbol transfer effects. Journal of Experimental Psychology. Learning, Memory, and Cognition, 20, 1139-1153.

Rouder, J. N., \& Morey, R. D. (2011). A Bayes meta-analysis of BEM's ESP claim. Psychonomic Bulletin \& Review, 18, 682-689.

Rusconi, E., Galfano, G., Rebonato, E., \& Umiltà, C. (2006). Bidirectional links in the network of multiplication facts. Psychological Research, 70, 32-42.

Wagenmakers, E.-J., Wetzels, R., Borsboom, D., \& van der Maas, H. (2011). Why psychologists must change the way they analyze their data: The case of psi: Comment on Bem (2011). Journal of Personality and Social Psychology, 100, 426-432.

Zbrodoff, N. J., \& Logan, G. D. (2005). What everyone finds: The problems-size effect. In J. I. D. Campbell (Ed.), Handbook of mathematical cognition (pp. 331-345). New York: Psychology Press. 